\title{
Managing antimicrobial resistance requires resisting the inappropriate use of antimicrobials
}

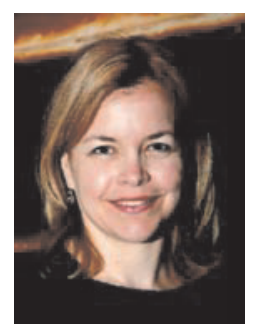

Susan Maddocks Deputy Edito

smaddocks@ mja.com.au

Competing interests: I am a member of ASID.

doi: 10.5694/mjal2.c0319

MJA News and

Careers Editor

smcnamara@

mja.com.au

Careers follows $p 360$ $\mathrm{n}$ this issue of the MJA, we are pleased to publish a collection of papers to be presented at the upcoming

Australasian Society for Infectious Diseases (ASID) scientific meeting.

In their editorial (page 292), the current President of ASID, Thomas Gottlieb, and colleagues declare antimicrobial resistance to be a major challenge for the management of infectious diseases in Australia over the next decade and call for the development of a national "antimicrobial resistance management body" as part of an Australian Centre for Disease Control.

Commons and colleagues (page 332) describe how the rapidly developing resistance of Salmonella spp overseas has affected our antibiotic choices for managing enteric fever in returning travellers. Gubler (page 293) reminds us that new epidemics are likely to arise within our own Asia-Pacific region, and Collignon and Page (page 302) debate the potential effects on human health of the use of antibiotics in farmed animals.

On page 327, Lim and colleagues describe the use of antimicrobials in four residential aged care facilities (RACFs) and find that $40 \%$ of antimicrobial courses are given for symptoms that do not fulfill criteria for infection, while more than $60 \%$ of infections have no specimens collected for diagnosis.

Herein lies a core dilemma in managing local antimicrobial resistance: the mismatch between ideal infection management and the reality of day-to-day infection management.
Ideally, when infection is suspected, a diagnostic specimen is taken and microbiological confirmation guides prescription of targeted, narrow-spectrum antimicrobial therapy. In reality, an often time-pressed doctor, when faced with a confused elderly patient with possible infection, knows that deterioration could be rapid and perhaps fatal, staffing - and therefore observation of the patient - may not be optimal, results for specimens will take time to come back, and there is an expectation that "something" be done. Prescribing a broad-spectrum antimicrobial in this situation may seem practical and sensible. Unfortunately, this is more likely to promote resistance development. This scenario is not peculiar to RACFs, but occurs in hospitals and in community practice.

The question is how to reconcile the practical with the ideal. Ideal prescribing, for a treating doctor, would require the availability of rapid diagnostic testing and local antimicrobial stewardship programs that use periodic surveillance of microbial resistance within the institution to guide antimicrobial choice.

More generally, to achieve improved infection control, we need enhanced collaboration with our Asia-Pacific neighbours, incorporating surveillance, collaborative infection control guidelines and epidemic response plans; and we need improved strategies to deal with antimicrobial use in the farming sector locally and globally.

In practice, however, it behoves all of us to consider the possibility of a future without effective antimicrobials every time we choose to prescribe one, and to pause - and, perhaps ... resist.

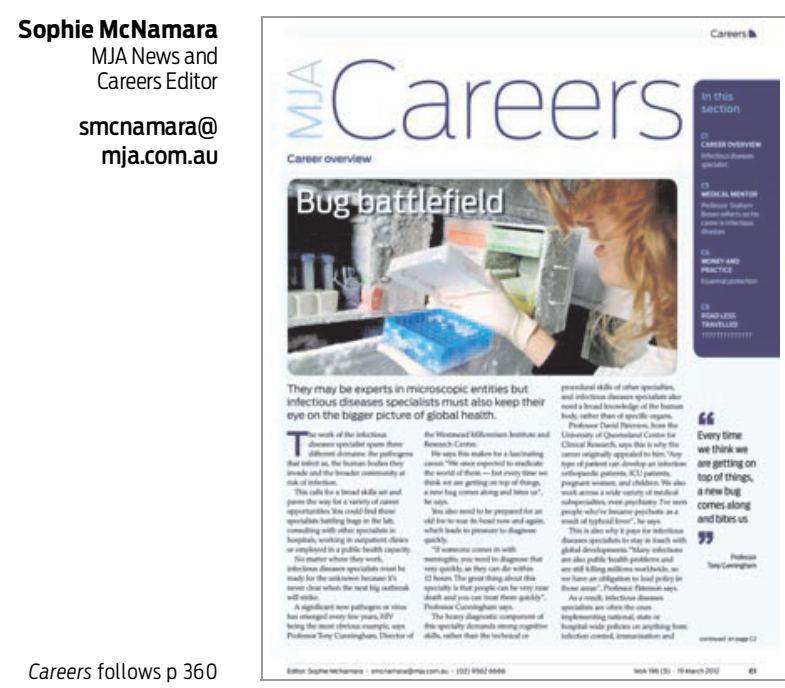

\section{Are you ready?}

It's the old cliche: doctors look after everyone else's health, but neglect their own. In this issue of MJA Careers, a radiologist who was recently diagnosed with nonHodgkin lymphoma gives his practical advice on how to be prepared in case of a major illness (page C9). Also, in the regular Money and Practice section, we talk to experts about ensuring you're set up financially if you suffer a major illness or accident (page C6). And while the rest of the Journal focuses on advances in infectious diseases, the Careers section looks at what it is really like to work as an infectious diseases physician. Turn to page C1, at the back of the Journal, to see what prominent infectious diseases specialists love about their job. 